\title{
BISEPARATING LINEAR MAPS BETWEEN CONTINUOUS VECTOR-VALUED FUNCTION SPACES
}

\author{
HWA-LONG GAU, JYH-SHYANG JEANG and NGAI-CHING WONG
}

(Received 15 January 2001; revised 8 November 2001)

Communicated by A. Pryde

\begin{abstract}
Let $X, Y$ be compact Hausdorff spaces and $E, F$ be Banach spaces. A linear map $T: C(X, E) \rightarrow C(Y, F)$ is separating if $T f, T g$ have disjoint cozeroes whenever $f, g$ have disjoint cozeroes. We prove that a biseparating linear bijection $T$ (that is, $T$ and $T^{-1}$ are separating) is a weighted composition operator $T f=h \cdot f \circ \varphi$. Here, $h$ is a function from $Y$ into the set of invertible linear operators from $E$ onto $F$, and $\varphi$ is a homeomorphism from $Y$ onto $X$. We also show that $T$ is bounded if and only if $h(y)$ is a bounded operator from $E$ onto $F$ for all $y$ in $Y$. In this case, $h$ is continuous with respect to the strong operator topology.
\end{abstract}

2000 Mathematics subject classification: primary 47B33, 47B38.

\section{Introduction}

Let $X$ and $Y$ be compact Hausdorff spaces, $E$ and $F$ be Banach spaces, and $C(X, E)$ and $C(Y, F)$ be the Banach spaces of continuous $E$-valued and $F$-valued functions defined on $X$ and $Y$, respectively. In $C(X, E)$, we write $f g=0$ for $\|f(x)\|\|g(x)\|=$ 0 , for every $x \in X$. A linear operator $T: C(X, E) \rightarrow C(Y, F)$ is said to be separating, or (cozero) disjointness preserving, if $T f T g=0$ whenever $f g=0$. An invertible $T$ is biseparating if both $T$ and $T^{-1}$ are separating.

The notion of disjointness preserving operators seems to be used firstly in the 40's (see for example $[25,26]$ ). Since then many authors have developed this concept in different directions. Abramovich, for example, made many contributions in the context

Research partially supported by the National Science Council of the Republic of China.

(C) 2003 Australian Mathematical Society 1446-7887/03 \$A2.00+0.00 
of Banach and vector lattices (see for example $[4,5]$ ). In the case of continuous scalarvalued functions, separating linear maps were studied by Beckenstein and Narici in [13-15] and further investigated in [10] and [19]. Separating linear bijections between spaces of continuous functions are automatically continuous. Indeed, a bijective linear operator $T$ from $C(X)$ onto $C(Y)$ is separating if and only if $T$ is an (automatically bounded) weighted composition operator (see for example [17, 19,21]). This can be considered as a special case of the generalized Nakano's theorem obtained in $[5,6]$, which asserts that d-isomorphic Banach lattices are order isomorphic and the $\mathrm{d}$-isomorphism is continuous. We recall that a d-isomorphism $T$ between Banach lattices $E$ and $F$ is a disjointness preserving linear bijection, and disjointness of elements $x, y$ in a Banach lattice is defined by $|x| \wedge|y|=0$. In this case, $E$ and $F$ are said to be d-isomorphic. Moreover, the inverse of a d-isomorphism is again a d-isomorphism, and positive d-isomorphisms are exactly lattice isomorphisms (see for example [7]). In the context of vector-valued functions, however, a separating or even a biseparating linear operator is not necessarily continuous (see Example 2.4).

In [23], Jerison got the first vector-valued version of the Banach-Stone Theorem: If $E$ is a strictly convex Banach space then every surjective isometry $T$ from $C(X, E)$ onto $C(Y, E)$ can be written as a weighted composition operator $T f=h \cdot f \circ \varphi$, that is,

$$
T f(y)=h(y)(f(\varphi(y))), \quad \forall y \in Y, \forall f \in C(X, E) .
$$

Here, $\varphi$ is a homeomorphism from $Y$ onto $X$ and $h$ is a continuous map from $Y$ into the space $(B(E, E)$, SOT) of bounded linear operators from $E$ into $E$ equipped with the strong operator topology (SOT). Moreover, $h(y)$ is an isometry from $E$ onto $E$ for all $y$ in $Y$. The conclusion might not hold, however, if $E$ is not strictly convex (see [20]).

After Jerison [23], many authors worked on different variants of the vector-valued Banach-Stone Theorem (see [12, 16, 18, 20,22-24]). In particular, as an extension of the representation theorem of Abramovich [1], Hernandez, Beckenstein and Narici proved in [12] that if $T$ is an isometric biseparating linear map from $C(X, E)$ onto $C(Y, F)$ then $T$ is a weighted composition operator $T f(y)=h(y)(f(\varphi(y)))$. In the case that $T$ is bounded but not necessarily invertible, $T$ can still be written as a weighted composition operator (see $[16,18]$ ). It is then possible to prove that every bounded biseparating linear map provides a homeomorphism $\varphi$ from $Y$ onto $X$. However, these methods might not apply to unbounded biseparating linear maps.

In Section 2, we develop a new argument to prove that every biseparating linear bijection $T$ from $C(X, E)$ onto $C(Y, F)$ induces a homeomorphism $\varphi$ from $Y$ onto $X$. As expected, $T$ is a weighted composition operator

$$
T f(y)=h(y)(f(\varphi(y))), \quad \forall f \in C(X, E), \forall y \in Y .
$$


Here, $h(y)$ is an invertible linear map from $E$ onto $F$ for each $y$ in $Y$. However, $T$ is not necessarily bounded (see Example 2.4). In fact, $T$ is bounded if and only if $\|h(y)\|<\infty$ for all $y$ in $Y$. In this case, $h: Y \rightarrow(B(E, F)$, SOT $)$ is continuous and $\|T\|=\sup _{y \in Y}\|h(y)\|<\infty$.

In the last section we discuss when the inverse $T^{-1}$ of a disjointness preserving linear bijection $T: C(X, E) \rightarrow C(Y, F)$ is disjointness preserving. It is known that $T^{-1}$ is disjointness preserving when $E=F$ is the scalar field (see for example [11]). However, this is not the case even for finite dimensional $E$ and $F$ (see Example 3.1; see also [2-4]). We will present a new condition, so-called (support) containment preserving property of $T$, which is originally due to Abramovich and Kitover [3]. We prove that $T^{-1}$ preserves disjointness if $T$ preserves containment. In particular, $T$ preserves both disjointness and containment if and only if it is so for $T^{-1}$ which happens if and only if $T$ and $T^{-\boldsymbol{P}}$ are both weighted composition operators. This can be considered as the vector-valued version of the results in [3].

We would like to express our deep thanks to Yuri Abramovich and the referee for several improvements and corrections.

\section{Biseparating linear maps are weighted composition operators}

In the following, we always assume $X$ and $Y$ are compact Hausdorff spaces, $E$ and $F$ are Banach spaces, and $B(E, F)$ is the space of bounded linear operators from $E$ into $F$ equipped with the strong operator topology. For each $x$ in $X$, let

$$
I_{x}=\{f \in C(X, E): f \text { vanishes in a neighbourhood of } x\} .
$$

Note that the linear manifold $I_{x}$ is not in general closed. But it is dense in the closed linear subspace $M_{x}=\{f \in C(X, E): f(x)=0\}$. Moreover, it is somehow 'prime' in the sense that $f \in I_{x}$ whenever $f g=0$ and $g(x) \neq 0$. In fact, $\|g(y)\|>0$ for all $y$ in a neighbourhood $V$ of $x$ and this forces $f$ to vanish in $V$.

We start by observing that a biseparating linear bijection $T$ preserves $I_{x}$ 's.

LEMMA 2.1. Let $T: C(X, E) \rightarrow C(Y, F)$ be a biseparating linear bijection. Then for each $x$ in $X$ there is a unique $y$ in $Y$ such that $T I_{x}=I_{y}$. Moreover, this defines a bijection $\varphi$ from $Y$ onto $X$ by $\varphi(y)=x$.

PROOF. For each $x$ in $X$, denote by $\operatorname{ker} T\left(I_{x}\right)$ the set $\bigcap_{f \in I_{x}}(T f)^{-1}(0)$. We first claim that $\operatorname{ker} T\left(I_{x}\right)$ is non-empty. Suppose on the contrary that for each $y$ in $Y$, there were an $f_{y}$ in $I_{x}$ with $T f_{y}(y) \neq 0$. Thus, an open neighbourhood $U_{y}$ of $y$ exists such that $T f_{y}$ is nonvanishing in $U_{y}$. Since $Y=\bigcup_{y \in Y} U_{y}$ and $Y$ is compact, $Y=U_{y_{1}} \cup U_{y_{2}} \cup \ldots \cup U_{y_{n}}$ for some $y_{1}, y_{2}, \ldots, y_{n}$ in $Y$. Let $V$ be an open neighbourhood 
of $x$ such that $\left.f_{y_{i}}\right|_{v}=0$ for all $i=1,2, \ldots, n$. Let $g \in C(X, E)$ such that $g(x) \neq 0$ and $g$ vanishes outside $V$. Then $f_{y_{i}} g=0$, and thus $T f_{y_{i}} T g=0$ since $T$ preserves disjointness. This forces $\left.T g\right|_{U_{i}}=0$ for all $i=1,2, \ldots, n$. Therefore, $T g=0$ and hence $g=0$ by the injectivity of $T$, a contradiction. We thus prove that $\operatorname{ker} T\left(I_{x}\right) \neq \emptyset$.

Let $y \in \operatorname{ker} T\left(I_{x}\right)$. For each $f \in I_{x}$, we want to show that $T f \in I_{y}$. If there exists a $g$ in $C(X, E)$ such that $T g(y) \neq 0$ and $f g=0$, then we are done by the disjointness preserving property of $T$. Suppose there were no such $g$; that is, for any $g$ in $C(X, E)$ vanishing outside $V=f^{-1}(0)$, we have $T g(y)=0$. Let $W \subset V$ be a compact neighbourhood of $x$ and $k \in C(X)$ such that $\left.k\right|_{W}=1$ and $k$ vanishes outside $V$. Then for any $g$ in $C(X, E), g=k g+(1-k) g$. Since $\left.(1-k)\right|_{W}=0$, we have $(1-k) g \in I_{x}$. This implies $T((1-k) g)(y)=0$ as $y \in \operatorname{ker} T\left(I_{x}\right)$. On the other hand, $\mathrm{kg}$ vanishes outside $V$. Hence $T(\mathrm{~kg})(y)=0$ by the above assumption. It follows that $T g(y)=T k g(y)+T((1-k) g)(y)=0$ for all $g$ in $C(X, E)$. This conflicts with the surjectivity of $T$. Therefore, $T I_{x} \subseteq I_{y}$.

Similarly, $T^{-1}\left(I_{y}\right) \subseteq I_{x^{\prime}}$ for some $x^{\prime}$ in $X$ since $T^{-1}$ is also separating. It follows that $I_{x} \subseteq T^{-1}\left(I_{y}\right) \subseteq I_{x^{\prime}}$. Consequently, $x=x^{\prime}$ and $T\left(I_{x}\right)=I_{y}$. The bijectivity of $\varphi$ is also clear now.

THEOREM 2.2. Two compact Hausdorff spaces $X$ and $Y$ are homeomorphic whenever there is a biseparating linear bijection $T$ from $C(X, E)$ onto $C(Y, F)$.

Proof. We show that the bijection $\varphi$ given in Lemma 2.1 is a homeomorphism. It suffices to verify the continuity of $\varphi$ since $Y$ is compact and $X$ is Hausdorff. Suppose, by the compactness of $X$, that there exists a net $\left\{y_{\lambda}\right\}_{\lambda}$ in $Y$ converging to $y$ but $\varphi\left(y_{\lambda}\right) \rightarrow x \neq \varphi(y)$, and we want to derive a contradiction.

Let $U_{x}$ and $U_{\varphi(y)}$ be disjoint open neighbourhoods of $x$ and $\varphi(y)$, respectively. Now for any $f$ in $C(X, E)$ vanishing outside $U_{\varphi(y)}$, we shall show that $T f(y)=0$. In fact, $\varphi\left(y_{\lambda}\right)$ belongs to $U_{x}$ for large $\lambda$. Since $\left.f\right|_{U_{x}}=0$ and $U_{x}$ is also a neighbourhood of $\varphi\left(y_{\lambda}\right)$, we have $f \in I_{\varphi\left(y_{\lambda}\right)}$. By Lemma 2.1,Tf $\in I_{y_{\lambda}}$ and in particular $T f\left(y_{\lambda}\right)=0$ for large $\lambda$. This implies $T f(y)=0$ by the continuity of $T f$.

Let $k \in C(X)$ be such that $\left.k\right|_{v}=1$ and $k$ vanishes outside $U_{\varphi(y)}$, where $V \subset U_{\varphi(y)}$ is a compact neighbourhood of $\varphi(y)$. Then $g=k g+(1-k) g$ for every $g$ in $C(X, E)$. Since $k g$ vanishes outside $U_{\varphi(y)}$, we have $T(k g)(y)=0$. On the other hand, we have $(1-k) g \in I_{\varphi(y)}$ since $\left.(1-k)\right|_{v}=0$. By Lemma 2.1, $T((1-k) g) \in I_{y}$ and thus $T((1-k) g)(y)=0$. It follows that $T g(y)=T(k g)(y)+T((1-k) g)(y)=0$. This is a contradiction since $T$ is onto. Hence $\varphi$ is a homeomorphism.

THEOREM 2.3. Every biseparating linear bijection $T: C(X, E) \rightarrow C(Y, F)$ is a weighted composition operator

$$
T f(y)=h(y)(f(\varphi(y))), \quad \forall f \in C(X, E), \forall y \in Y .
$$


Here $\varphi$ is a homeomorphism from $Y$ onto $X$ and $h(y)$ is an invertible linear map from $E$ onto $F$ for each $y$ in $Y$. Moreover, $T$ is bounded if and only if $h(y)$ is bounded for all $y$ in $Y$. In this case, $h$ is a continuous map from $Y$ into $(B(E, F)$, SOT), and $\|T\|=\sup _{y \in Y}\|h(y)\|$.

Proof. By Theorem 2.2, we have a homeomorphism $\varphi$ from $Y$ onto $X$ such that $T\left(I_{x}\right)=I_{y}$ where $\varphi(y)=x$.

\section{Claim. $T M_{x} \subseteq M_{y}$.}

If the claim is verified, then $T M_{x}=M_{y}$ by the same argument for $T^{-1}$. It follows that $\operatorname{ker} \delta_{x}=\operatorname{ker} \delta_{y} \circ T$, where $\delta_{x}$ and $\delta_{y}$ are point evaluations at $x$ and $y$, respectively. Consequently, there is an invertible linear operator $h(y)$ from $E$ onto $F$ such that $\delta_{y} \circ T=h(y) \circ \delta_{x}$. Equivalently, $T f(y)=h(y)(f(\varphi(y)))$ for all $f$ in $C(X, E)$ and $y$ in $Y$.

Suppose $T$ is bounded. For any $e$ in $E$, let $f \in C(X, E)$ such that $f(x)=e, \forall x \in$ $X$. Since $\|h(y) e\|=\|h(y)(f(\varphi(y)))\|=\|T f(y)\| \leq\|T f\| \leq\|T\|\|f\|=\|T\|\|e\|$, we conclude that $\|h(y)\| \leq\|T\|$ for all $y$ in $Y$. On the other hand, assume $\|h(y)\|<\infty$ for all $y$ in $Y$. Let $\left\{y_{\lambda}\right\}_{\lambda}$ be a net converging to $y$ in $Y$. Then $\left\|h\left(y_{\lambda}\right) e-h(y) e\right\|=$ $\left\|h\left(y_{\lambda}\right)\left(f\left(\varphi\left(y_{\lambda}\right)\right)\right)-h(y)(f(\varphi(y)))\right\|=\left\|T f\left(y_{\lambda}\right)-T f(y)\right\| \rightarrow 0$ as $T f \in C(Y, F)$. Thus $h$ is continuous on $Y$. Moreover, $\sup _{y \in Y}\|h(y) e\|=\sup _{y \in Y}\|T f(y)\|<\infty$. Therefore, $\sup _{y \in Y}\|h(y)\|<\infty$ by the Principle of Uniform Boundedness. Finally, for any $g$ in $C(X, E)$ and $y$ in $Y$, we have $\|T g(y)\|=\|h(y)(g(\varphi(y)))\| \leq\|h(y)\|\|g\|$. Hence $\|T\| \leq \sup _{y \in Y}\|h(y)\|$.

To verify the claim, suppose on the contrary $f \in M_{x}$ but $T f(y) \neq 0$. If $x$ belongs to the interior of $f^{-1}(0)$, then $f \in I_{x}$ and thus $T f(y)=0$. Therefore, we may assume there is a net $\left\{x_{\lambda}\right\}_{\lambda}$ in $X$ converging to $x$ and $f\left(x_{\lambda}\right)$ is never zero. Let $y_{\lambda}$ be in $Y$ such that $\varphi\left(y_{\lambda}\right)=x_{\lambda}$. Clearly, $y_{\lambda}$ converges to $y$ and we may assume there is a constant $\epsilon$ such that $\left\|T f\left(y_{\lambda}\right)\right\| \geq \epsilon>0$ for all $\lambda$. For $n=1,2, \ldots$, set

$$
V_{n}=\left\{z \in X: \frac{1}{2 n+1}<\|f(z)\|<\frac{1}{2 n}\right\}
$$

and

$$
W_{n}=\left\{z \in X: \frac{1}{2 n}<\|f(z)\|<\frac{1}{2 n-1}\right\} \text {. }
$$

Then at least one of the unions $V=\bigcup_{n=1}^{\infty} \bar{V}_{n}$ and $W=\bigcup_{n=1}^{\infty} \overline{W_{n}}$ contains a subnet of $\left\{x_{\lambda}\right\}_{\lambda}$. Without loss of generality, we assume that all $x_{\lambda}$ belong to $V$. Let $V_{n}^{\prime}$ be an open set containing the closure $\bar{V}_{n}$ of $V_{n}$ such that $V_{n}^{\prime} \cap V_{m}^{\prime}=\emptyset$ if $n \neq m$. Let $g_{n}$ in $C(X, E)$ be of norm at most $1 / 2 n$ such that $g_{n}$ agrees with $f$ on $V_{n}$ and vanishes outside $V_{n}^{\prime}$ for each $n$. Then $g_{n} g_{m}=0$ for all $m \neq n$. Let $g(w)=\sum_{n=1}^{\infty} \sqrt{2 n} g_{n}(w)$, 
for every $w \in X$. Since for each $w$ in $X$ at most one term in the above sum is nonzero and $\left\|\sqrt{2 n} g_{n}\right\| \leq 1 / \sqrt{2 n}$, the convergence is uniform and thus $g \in C(X, E)$. Note that for each $n, g$ agrees with $\sqrt{2 n} f$ on each open set $V_{n}$. Moreover, each $x_{\lambda}$ belongs to a unique $V_{n(\lambda)}$ and $n(\lambda) \rightarrow \infty$ as $\lambda \rightarrow \infty$. Therefore, $g-\sqrt{2 n(\lambda)} f \in I_{x_{\lambda}}$. This implies $T(g-\sqrt{2 n(\lambda)} f) \in I_{y_{\lambda}}$ and thus $\left\|T g\left(y_{\lambda}\right)\right\|=\sqrt{2 n(\lambda)}\left\|T f\left(y_{\lambda}\right)\right\| \geq \sqrt{2 n(\lambda)} \epsilon \rightarrow \infty$ as $\lambda \rightarrow \infty$. But the limit should be the finite number $\|T g(y)\|$, a contradiction. This completes the proof.

In the following example, we see that the invertible linear operator $h(y)$ in (1) can be unbounded.

EXAMPLE 2.4. Let $X=\{0\}$ and $\psi$ be an unbounded linear functional of $c_{0}$ such that $\psi((1,0,0, \cdots))=1$. Define an unbounded linear bijection $H$ from $c_{0}$ onto $c_{0}$ by

$$
H(\lambda)=\left(\lambda_{1}+\psi(\lambda), \lambda_{2}, \lambda_{3}, \cdots\right), \quad \forall \lambda=\left(\lambda_{1}, \lambda_{2}, \lambda_{3}, \cdots\right) \in c_{0} .
$$

Set $h(0)=H$ and define the biseparating linear bijection $T: C\left(X, c_{0}\right) \rightarrow C\left(X, c_{0}\right)$ by

$$
T f(0)=h(0)((f(0))), \quad \forall f \in C\left(X, c_{0}\right) .
$$

Then $T$ is an unbounded weighted composition operator. Note that $\varphi: X \rightarrow X$ with $\varphi(0)=0$ is a homeomorphism.

REMARK. Recently, Araujo has discussed related problems in $[8,9]$. For example, he proved independently that two completely regular spaces $X$ and $Y$ have homeomorphic realcompactifications $v X$ and $v Y$ whenever there is a biseparating linear maps from the space $C_{b}(X, E)$ of bounded continuous vector-valued functions defined on $X$ onto the space $C_{b}(Y, F)$ of such defined on $Y$, where $E$ and $F$ are Banach spaces. This will give similar results to those in this section. However, the approach in $[8,9]$ is somewhat different from our methods and it has stronger topological flavour, while the current paper emphasizes the algebraic aspects of the problem. In particular, we consider the zero preserving property of a linear biseparation (Lemma 2.1) whilst Araujo and some other authors utilize notions of supporting functionals and peak points. In this sense, we provide not only new results but also new techniques, which we think are useful additions to the literature.

\section{Containment and disjointness preserving operators}

For the results of the preceding section we had to assume that $T$ is biseparating, namely that both $T$ and $T^{-1}$ are separating. It is known that the inverse of a separating 
linear bijection between Banach lattices (in particular, $C(X)$ 's) always preserves disjointness (see for example [2, Theorem 1]). Recently, Abramovich and Kitover $[2,4]$ showed that $T^{-1}$ need not be separating in the general vector lattice setting.

EXAMPLE $3.1([12])$. Let $X=\{0\}$ and $E=\mathbb{R}^{2}$ with sup norm, and let $Y=\{1,2\}$ and $F=\mathbb{R}$ with its usual norm. Define $T: C(X, E) \rightarrow C(Y, F)$ by $T\left(\begin{array}{l}a \\ b\end{array}\right)=g$ with $g(1)=a$ and $g(2)=b$. Then the surjective linear isometry $T$ is separating, but its inverse $T^{-1}$ is not.

Recall that for an $f$ in $C(X, E)$, the cozero of $f$ is $\operatorname{coz}(f)=\{x \in X: f(x) \neq 0\}$ and the support $\operatorname{supp}(f)$ of $f$ is the closure of $\operatorname{coz}(f)$ in $X$. The following definition modifies the one given by Abramovich [3].

Definition 3.2. A linear map $T: C(X, E) \rightarrow C(Y, F)$ is said to be (support) containment preserving if $\operatorname{supp}(f) \subseteq \operatorname{supp}(g)$ implies $\operatorname{supp}(T f) \subseteq \operatorname{supp}(T g)$.

For any injective map $T: C(X, E) \rightarrow C(Y, F)$ we denote by $T^{-1}$ the inverse operator defined on ran $T$, the range space of $T$.

LEMMA 3.3. Let $T: C(X, E) \rightarrow C(Y, F)$ be a linear injection. If $T$ is containment preserving, then $T^{-1}$ is disjointness preserving.

Proof. Suppose, on the contrary, that there exist $f$ and $g$ in $C(X, E)$ such that Tf $T g=0$ but $\|f(x)\|\|g(x)\| \neq 0$ for some $x$ in $X$. Then we can find an open neighbourhood $V$ of $x$ such that $V \subset \operatorname{coz}(f) \cap \operatorname{coz}(g)$. Let $h \in C(X, E)$ such that $h(x) \neq 0$ and $\left.h\right|_{v^{c}}=0$. It is clear that $\operatorname{supp}(h) \subseteq \operatorname{supp}(f) \cap \operatorname{supp}(g)$. Since $T$ preserves containment, we have $\operatorname{supp}(T h) \subseteq \operatorname{supp}(T f) \cap \operatorname{supp}(T g)$. On the other hand, $T f T g=0$ implies $\operatorname{coz}(T f) \cap \operatorname{coz}(T g)=\emptyset$. It follows that $\operatorname{coz}(T h) \subseteq$ $\operatorname{supp}(T f) \subseteq Y \backslash \operatorname{coz}(T g)$. Since $\operatorname{coz}(T h)$ is open, it forces that $\operatorname{coz}(T h) \cap \operatorname{supp}(T g)=$ $\emptyset$. Therefore, $T h=0$ and hence $\cdot h=0$ by the injectivity of $T$, a contradiction. We thus prove that $T^{-1}$ is disjointness preserving.

Combining Theorem 2.3 and Lemma 3.3, we will have the following

COROLlary 3.4. Let $T: C(X, E) \rightarrow C(Y, F)$ be a linear bijection. Then the following statements are equivalent

(a) $T$ preserves disjointness and containment;

(b) $T$ and $T^{-1}$ preserve disjointness;

(c) $T$ and $T^{-1}$ preserve containment;

(d) $T$ and $T^{-1}$ are weighted composition operators. 
Proof. We need only to prove (b) implies (a). Suppose $\operatorname{supp}(f) \subseteq \operatorname{supp}(g)$, we want to show that $\operatorname{supp}(T f) \subseteq \operatorname{supp}(T g)$. Suppose on the contrary that there exists $y$ in $Y$ such that $y \in \operatorname{supp}(T f) \backslash \operatorname{supp}(T g)$. Thus, there exists an open neighbourhood $V$ of $y$ such that $V \cap \operatorname{supp}(T g)=\emptyset$. Choose $y^{\prime} \in V \cap \operatorname{coz}(T f)$ and let $h \in C(Y, F)$ such that $h\left(y^{\prime}\right) \neq 0$ and $\left.h\right|_{v^{c}}=0$. Since $T$ is surjective, say $T k=h$ for some $k$ in $C(X, E)$. Then $T k T g=0$, and thus $k g=0$ since $T^{-1}$ preserves disjointness. This forces $k f=0$, because $\operatorname{supp}(f) \subseteq \operatorname{supp}(g)$. Therefore, $h T f=T k T f=0$. But $\left\|h\left(y^{\prime}\right)\right\|\left\|T f\left(y^{\prime}\right)\right\| \neq 0$, a contradiction.

In Example 3.1, the surjective isometry $T$ preserves disjointness but not containment while its inverse $T^{-1}$ preserves containment but not disjointness. Moreover, $T$ is a weighted composition operator but $T^{-1}$ is not.

\section{References}

[1] Y. A. Abramovich, 'Multiplicative representation of operators preserving disjointness', Netherl. Acad. Wetensch. Proc. Ser. A 86 (1983), 265-279.

[2] Y. A. Abramovich and A. K. Kitover, 'A solution to a problem on invertible disjointness preserving operators', Proc. Amer. Math. Soc. 126 (1998), 1501-1505.

[3] _ - 'A characterization of operators preserving disjointness in terms of their inverse', Positivity $4(2000), 205-212$.

[4] - Inverses of disjointness preserving operators, Mem. Amer. Math. Soc. 143 (Amer. Math. Soc., Providence, 2000).

[5] Y. A. Abramovich, A. I. Veksler and A. V. Koldunov, 'Operators preserving disjointness', Dokl. Akad. Nauk USSR 248 (1979), 1033-1036.

[6] —, 'Operators preserving disjointness, their continuity and multiplicative representation', in: Linear Operators and Their Applications, Sb. Nauchn. Trudov (Leningrad, 1981) pp. 13-34.

[7] C. D. Aliprantis and O. Burkinshaw, Positive operators (Academic Press, Orlando, 1985).

[8] J. Araújo, 'Realcompactness and Banach-Stone theorems', preprint http: / / xxx. lanl.gov/paf/math/0010292.

[9] — ' 'Realcompactness and spaces of vector-valued functions', preprint http://xxx. Ianl.gov/pdf/math/0010261.

[10] J. Araújo, E. Beckenstein and L. Narici, 'Biseparating maps and ring of continuous functions', in: Proceedings of the Eighth Summer Conference on Topology, Queens College, New York, June 1992, Ann. New York Acad. Sci. 728 (New York Acad. Sci., New York, 1994) pp. 296-303.

[11] E. Beckenstein, J. Araújo and L. Narici, 'When is a separating map biseparating?', Arch. Math. (Basel) 67 (1996), 395-407.

[12] E. Beckenstein, S. Hernández and L. Narici, 'Banach-Stone theorems and separating maps', Manuscripta Math. 86 (1995), 409-416.

[13] E. Beckenstein and L. Narici, 'Automatic continuity of certain linear isomorphism', Acad. Roy. Belg. Bull. Cl. Sci. (5) 73 (1987), 191-200.

[14] _ 'A nonarchimedean Stone-Banach theorem', Proc. Amer. Math. Soc. 100 (1987), 242-246.

[15] - 'Automatic continuity of linear maps of spaces of continuous functions', Manuscripta Math. 62 (1988), 257-275.

[16] J. T. Chan, 'Operators with the disjoint support property', J. Operator Theory 24 (1990), 383-391. 
[17] J. J. Font and S. Hernández, 'On separating maps between locally compact spaces', Arch. Math. (Basel) 63 (1994), 158-165.

[18] J. E. Jamison and M. Rajagopalan, 'Weighted composition operator on $C(X, E)$ ', J. Operator Theory 20 (1988), 307-317.

[19] K. Jarosz, 'Automatic continuity of separating linear isomorphisms', Canad. Math. Bull. 33 (1990), $139-144$.

[20] J. S. Jeang and N. C. Wong, 'On the Banach-Stone problem', preprint.

[21] - 'Weighted composition operators of $C_{0}(X)$ 's', J. Math. Anal. Appl. 201 (1996), 981-993.

[22] —_, 'Into isometries of $C_{0}(X, E)$ 's', J. Math. Anal. Appl. 207 (1997), 286-290.

[23] M. Jerison, 'The space of bounded maps into a Banach space', Ann. of Math. 52 (1950), 309-327.

[24] K. S. Lau, 'A representation theorem for isometries of $C(X, E)$ ', Pacific J. Math. 60 (1975), 229-233.

[25] B. Z. Vulikh, 'On linear multiplicative operations', Dokl. Akad. Nauk USSR 41 (1943), 148-151.

[26] _ 'The product in linear partially-ordered spaces and its application to the theory of operations II', (Russian) Mat. Sb. N.S. 22 (1948), 267-317.

Department of Mathematics

National Central University

Chung-Li

Taiwan 320

R.O.C.

e-mail: hlgau@math.ncu.edu.tw
Department of Applied Mathematics

National Sun Yat-sen University

Kaohsiung

Taiwan 804

R.O.C.

e-mail: jeangjs@math.nsysu.edu.tw wong@math.nsysu.edu.tw 
